\title{
Passive resonance frequencies of flute structures
}

William J. Strong, Neville H. Fletcher, and Ron K. Silk

Citation: The Journal of the Acoustical Society of America 71, S90 (1982); doi: 10.1121/1.2019630

View online: https://doi.org/10.1121/1.2019630

View Table of Contents: https://asa.scitation.org/toc/jas/71/S1

Published by the Acoustical Society of America

\section{ARTICLES YOU MAY BE INTERESTED IN}

A study of flute control parameters

The Journal of the Acoustical Society of America 123, 3124 (2008); https://doi.org/10.1121/1.2933050

Acoustical analysis of the Boehm flute

The Journal of the Acoustical Society of America 65, 499 (1979); https://doi.org/10.1121/1.382350

Sound production in recorderlike instruments. I. Dimensionless amplitude of the internal acoustic field The Journal of the Acoustical Society of America 101, 2914 (1997); https://doi.org/10.1121/1.418521

Acoustics of the flute

Physics Today 21, 25 (1968); https://doi.org/10.1063/1.3034586 
plate calculated in this fashion is useful in statistical energy analysis applications to complex structures.

$4: 20$

RR8. SEA equivalences between large platelike systems and their subparts. Nancy S. Timmerman (Bolt Beranek and Newman Inc., 50 Moulton Street, Cambridge, MA 02238)

A procedure was developed to equate the properties of a large system, which is composed of a number of plates connected to each other in a variety of ways, with the properties of the smaller plates for use in Statistical Energy Analysis (SEA) modeling. Each small subsystem is a single plate, which can be characterized by a uniform thickness, an area, a material, and some internal losses. Each connection between systems is defined by a junction, with a length, a number of systems connected at that junction, and thicknesses of each system connected at that junction. Equivalents thicknesses, areas, loss factors, and junction lengths were determined for the larger composite system. The analysis was extended to the case where two composite systems are connected to each other.

\section{4:35}

RR9. Radiation from cylindrical shells at the neighborhood of the ring frequency. Azriel Harari (Code 3635, Naval Underwater Systems Center, Newport, RI 02840)

For many submerged cylindrical vehicles, there is a marked increase in the acoustical radiation from the structure at the neighborhood of the ring frequency. Several aspects of the shell dynamic characteristics that relate to radiated noise at the neighborhood of the ring frequency are considered here. The ratio between the wavelength of the shell and the wavelength of an acoustic fluid, the admittance level of an infinitely long cylindrical shell, the energy propagation along the cylindrical shell, and the mode shape of a finite cylindrical shell with flexible ends are studied.

THURSDAY AFTERNOON, 29 APRIL 1982

ROOM 8, 2:00 TO 5:00 P.M.

\title{
Session SS. Musical Acoustics VII: Wind Instruments and General
}

\author{
Stephen C. Thompson, Chairman \\ Gould Inc., Ocean Systems Division, 18901 Euclid Avenue, Cleveland, Ohio 44117
}

Chairman's Introduction-2:00

Invited Paper

2:05

SS1. Institute de Recherche et Coordination Acoustique/Musique-A guided tour. W. M. Hartmann and J.-P. Armand (IRCAM, 31 rue Saint-Merri, 75004 Paris, France)

The Institute for Research and Coordination of Acoustics and Music, directed by Pierre Boulez, pursues new ideas and techniques in music with the aid of acoustic and information science. IRCAM has personnel and facilities in place throughout the large terrain that concerns itself with the creation of musical sounds. There is research in psychoacoustics and in the acoustics of the human voice, of musical instruments and of rooms. Much of this research is of an applied nature, addressing the needs of particular compositions or performance situations. Acousticians often collaborate closely with individual musicians from the Ensemble Intercontemporain, an ensemble of professional musicians dedicated to comtemporary music, allied with the Institute. IRCAM contributes significantly to the continuing development of digital hardware and software for the analysis and synthesis of sounds. Most notable are IRACAM's high-speed digital synthesizers, which permit real-time control in the creation and modification of musical sounds. Musical acitivity at IRCAM extends from composition to performance and commercial recording. The Institute is host to composers from many countries, who employ its facilities to create electronic music. Performance and recording capabilities are greatly enhanced, by the Espace de Projection, an acoustically variable room of unparalleled flexibility. The present talk is a guided tour of IRCAM's unique laboratories and studios, with recorded examples of recent compositions.

\section{Contributed Papers}

\section{2:35}

SS2. Passive resonance frequencies of flute structures. William $\mathbf{J}$. Strong (Department of Physics and Astronomy, Brigham Young. University, Provo, UT 84602), Neville H. Fletcher, and Ron K. Silk (Department of Physics, University of New England, Armidale, NSW 2351, Australia)

Numerically calculated impedances for flute structures may be useful for exploring how changes in design parameters affect resonance frequencies. Any numerical method should be validated by comparing its results with experimental data. A successive impedance method was used to numerically calculate passive resonance frequencies from the dimensions of a Powell flute. Calculations were made for a given embouchure condition with different fingerings and for the middle and foot joints with different fingerings. The numerical results are compared to experimental results obtained for the same flute [John W. Coltman, J. Acoust. Soc. Am. 40, 99-107 (1966)]. The numerical results typically differ from the experimental data by a few cents, 
although some differ by as much as 20 cents. Similar comparisons are made for octave stretching with discrepancies of as much as 10 cents. [Work supported in part by Australian-American Educational Foundation.]

\section{2:50}

SS3. Second-harmonic sound generation at woodwind tone holes. W. B. Richards and A. H. Benade (Department of Physics, Case Western Reserve University, Cleveland, OH 44106)

Keefe has observed the importance of nonlinear processes in the tone-hole lattice of a woodwind instrument. Using a probe microphone, we have investigated second-harmonic generation in the neighborhood of the first open tone hole of two cylindrical air columns driven at $\mathbf{2 0 0}$ $\mathrm{Hz}$. The two lattices have tone holes with identical input impedance, but one has tone holes whose chimney height is small compared to the hole diameter, while the other has chimney height nearly the same as the hole diameter. When the SPL in the center of the bore opposite the tone hole is $107 \mathrm{~dB}$, the sound waves in the two tone-hole lattices have similar second-harmonic content, $-26 \mathrm{~dB}$ relative to the level of the fundamental. (The second harmonic contributed by the driver is $36 \mathrm{~dB}$ below the level of the fundamental.) When the mid-bore SPL is $122 \mathrm{~dB}$, the second-harmonic level within and immediately outside the short-chimney hole rises to $-11 \mathrm{~dB}$ relative to the fundamental, while the tall-chimney tone hole is at $-17 \mathrm{~dB}$. (At this level, the second harmonic at the driver is still negligible.) Streaming from the tone hole is present in all measurements, but the $3-\mathrm{dB}$ width of the second-harmonic spectral component is less than $0.08 \mathrm{~Hz}$ at all SPL. The large second-harmonic component does not propagate away from the region of the tone hole: it drains energy from the fundamental mode of oscillation, but does not contribute to the sound field in the room nor to the operation of the flow-control valve at the top of the instrument. [Work supported by NSF.]

\section{3:05}

SS4. Vocal tract effects in wind instrument regeneration. A. H. Benade and P. L. Hoekje (Department of Physics, Case Western Reserve University, Cleveland, OH 44106)

Wind players are much concerned with vocal tract influences on tone production. Laboratory study has been inconclusive, in part from inadequate theoretical formulation. A reed valve is opened and closed by the pressure difference $\left(p_{m}-p_{M}\right)$ across it: $p_{m}$ in the instrument's mouthpiece, $p_{M}$ in the player's mouth. The reed transconductance $A$ $=\partial u / \partial\left(p_{m}-p_{M}\right)$ depends on its natural frequency $\omega_{r}$ and its damping, becoming large near $\omega_{r}$. The condition for oscillation is $A\left[\left(Z_{m}+Z_{M}\right) /\right.$ $\left\langle Z_{r}\right] \geq 1$. We note that the reed impedance $Z_{r}$ is paralleled by the sum of $Z_{m}$ and $Z_{M}$. Oscillation requires $\operatorname{Im}\left(Z_{m}+Z_{M}\right) \neq 0$, with sign depending on the low-frequency sign of $A$ (positive for woodwinds, negative for brasses); whence woodwinds play with $\omega \leq \omega_{r}$, brasses with $\omega \geq \omega_{r}$. These results extend to large amplitude (mode-coupled) regimes of oscillation in the familiar way. Available data show resonance bandwidths of $Z_{M}$ are much larger than those of $Z_{m}$ (with smaller magnitude fluctuations), explaining the difficulty of direct measurement of musical effects. During tonal startup on long instruments (e.g., horn, tuba), $Z_{m}$ changes as successive reflections build up the standing wave. By itself $Z_{m}$ does not generally satisfy the regeneration condition during onset, thus giving a crucial role to the vocal tract. Results of experiments in progress will be presented. [Work supported by NSF.]

\section{3:30}

SS5. Reynolds and Strouhal numbers in woodwind musical instruments. Douglas H. Keefe (Cambridge Collaborative, Inc., P.O. Box 74, Cambridge, MA 02142)

It is convenient to use dimensional analysis in investigating nonlinear effects in woodwind instrument air columns. One dimensionless quantity characterizing the flow is the Reynolds number. There is both a time-varying particle velocity as well as a time-independent streaming velocity within the woodwind, and the Reynolds number $\boldsymbol{R}$ defined in terms of the streaming velocity may be written as $R \simeq$ $d^{2} / \delta^{2}$. The time-varying particle displacement is $d$ and the viscous boundary layer thickness is $\delta$. $R \ll 1$ corresponds to the type of streaming first considered by Rayleigh, whereas the streaming at larger values of $R$ is more complicated. The Strouhal number $S$ associated with the oscillating flow and characteristic length $L$ is $S=L / d$. The criterion that the nonlinear convective acceleration term $(v \nabla v)$ in the NavierStokes equation be small relative to the linearized acceleration is that $S \gg 1$. The particle displacement has been estimated at a fortissimo dynamic level on a flute, clarinet, and alto saxophone by means of pressure level measurements at the first open tone hole and the known radiation impedance of the hole. The streaming Reynolds number is approximately 0.2 for the flute and in the range 9-20 for the clarinet and sax for low register tones at the frequency of the repetition rate. Both $R$ and $d$ rapidly decrease for higher frequency components. The particle displacement ranges from $0.1 \mathrm{~mm}$ for the flute to $1 \mathrm{~mm}$ for the clarinet and saxophone. The Strouhal number of interest is defined for a characteristic length $L$ on the order of the radius of curvature of the sharp corners within the woodwind bore; for definiteness, $I$ take $L=0.5 \mathrm{~mm}$. The resulting Strouhal number is of order 5 for the flute and 0.5 for the clarinet and saxophone. Since the Strouhal number is not large compared to unity, then the nonlinear effects are important near sharp corners within the bore and particularly so for the clarinet and sax.

SS6. Mutes of brass instruments-Experiments and calculations. Rene Causse and Benny Sluchin (IRCAM, 31 rue Saint-Merri, 75004, Paris, France)

We have performed precision measurements to determine the effects of various mutes on the input impedance, external averaged spectra, and radiation patterns of brass instruments. A straight mute introduces a parasitic impedance peak and causes frequency shifts in neighboring impedance peaks. For horns which are approximately conical (e.g., tuba) the parasitic peak lies near the lowest resonance. For horns which are approximately hyperbolic (e.g., trumpet and trombone) the parasite normally lies near the second resonance. With increasing penetration of the mute the parasite frequency decreases. In general, the parasite frequency varies as the inverse square root of the mute volume. A cup mute and a Harmon mute may introduce two parasitic peaks. These effects of a mute may be calculated by modeling both horn and mute as a series of truncated cones and computing the input impedance by an iterative procedure. Calculations to date are in reasonable agreement with experiment. The external spectrum of a muted instrument, averaged over radiation direction and playing frequency, shows up to six prominent minima. These correspond well with calculated impedance minima of the mute itself. The mute material may also significantly affect the radiated spectrum. We have, therefore, studied four geometrically identical 